\title{
Hinweise für das Dilemma der Gleichzeitigkeit in den Demokratien Mittel- und Osteuropas
}

Grotz, Florian und Ferdinand Müller-Rommel (Hrsg.): Regierungssysteme in Mittel- und Osteuropa. Die neuen EU-Staaten im Vergleich, VS Verlag für Sozialwissenschaften, Wiesbaden 2011, 379 Seiten, $€ 29,95$.

Florian Grotz und Ferdinand Müller-Rommel konzipieren ihren Sammelband über die Regierungssysteme der neuen EU-Mitgliedstaaten in Mittel- und Osteuropa (MOE) nicht als Sammlung von Länderstudien, sondern als systematischen Vergleich. Um dieses anspruchsund verdienstvolle Vorhaben zu realisieren, greifen sie auf Arend Lijpharts in der Vergleichenden Politikwissenschaft breit rezipierte Studie über die zwei „Grundmuster der Demokratie“ zurück. Zwar lassen sich die MOE-Demokratien in diese Muster nicht einordnen - was der theoretisch-methodologischen Kritik an dieser Typologie ein empirisches Argument hinzufügt. Die ihr zugrunde liegenden Merkmalsdimensionen eignen sich jedoch sehr gut, um den Aufbau des Sammelbands zu strukturieren. Regierungssysteme können damit im weiteren Sinne, das heißt als „sämtliche funktionsrelevanten Strukturen einer demokratischen Verfassungsordnung" (S. 13), analysiert werden.

Die Beiträge des Bandes sind entsprechend zu vier Gruppen gebündelt: Zunächst werden der Verfassungsrahmen (Astrid Lorenz) sowie die Arrangements der horizontalen (Timm Beichelt und Dorothea Keudel) und vertikalen (Martin Brusis) Gewaltenteilung analysiert, eingeleitet durch eine allgemeine Evaluierung des Konsolidierungsgrades der Demokratie (Wolfgang Merkel). In einer zweiten Gruppe finden sich Analysen der Wahl-, Parteien- und Verbändesysteme (Philipp Harfst, Guido Tiemann und Klaus Armingeon). Drittens werden das parlamentarische Entscheidungszentrum, das heißt die Binnenorganisation von Parlamenten (Silvia von Steinsdorff), die Bildung und Dauerhaftigkeit von Regierungen (Florian Grotz und Till Weber) sowie die Binnenstruktur der Kernexekutiven (Ferdinand MüllerRommel), untersucht. Eine vierte Gruppe von Beiträgen befasst sich mit Formen der direkten Demokratie (Uwe Wagschal), der Rolle von Verfassungsgerichten (Christoph Hönnige) sowie dem Einfluss der Europäischen Union auf die Demokratisierung in den untersuchten Ländern (Frank Schimmelfennig und Guido Schwellnus).

Die MOE-Länder haben eine späte und spezifische Demokratisierung erfahren, die durch die staatssozialistische Ausgangssituation und später auch den EU-Beitrittsprozess geprägt war. Daher stellt sich einerseits die Frage nach Gruppen-Gemeinsamkeiten im Unterschied zu westeuropäischen Demokratien, also nach substantiellen Begründungen für das Festhalten an der Konstruktion einer europäischen Subregion. Andererseits ist auch nach Anzeichen der Konvergenz dieser subregionalen Gruppe oder einzelner Länder mit den alten EUMitgliedstaaten zu suchen. In ihrer Gesamtheit arbeiten die Beiträge heraus, dass wesentliche institutionelle Grundsatzentscheidungen der frühen 1990er Jahre, wie etwa zugunsten von Einheitsstaaten und parlamentarischen Regierungssystemen, in allen Ländern gleichartig waren und auch Strukturen der demokratischen Willensbildung sowie der politischen Entscheidungszentren entstanden sind, die sich untereinander und im Vergleich mit Westeuropa generell ähneln. Dennoch bleibt die Varianz innerhalb der Subregion in vielen Strukturdimensionen erheblich. Die einzelnen Beiträge qualifizieren diesen scheinbar trivialen Gesamtbefund hochinformativ, konzentriert und empirisch gut belegt. 
Differenziert fallen auch Befunde über die erreichte Annäherung an westliche Demokratien aus. So konstatiert beispielsweise Harfst (im Zeitverlauf nachlassende) Gemeinsamkeiten im Design der Wahlsysteme innerhalb der Subregion sowie die im Vergleich mit etablierten Demokratien (im Zeitverlauf zunehmende) Gleichheit der Wirkungen von Wahlsystemen auf Parteiensysteme, was letztlich deren Konsolidierung anzeigt. Tiemann findet einen Konvergenztrend zwischen den Parteiensystemen des „alten“ und „neuen“ Europa bei gleichwohl ausgeprägter intra-(sub)regionaler Varianz. Am deutlichsten und langfristigsten erscheinen Besonderheiten, die subregionale Gruppen-Unterschiede abbilden, bei Parteien und Verbänden, in der soziodemografischen Zusammensetzung der Parlamente und ihrer Binnenorganisation, bei der Regierungsbildung und -stabilität und in der hohen Fluktuation des politischen Personals. Bemerkenswerterweise sind direktdemokratische Verfahren in einigen Ländern verbreiteter als in Westeuropa. Verfassungsgerichte verfügen in allen MOE-Ländern über eine stärkere Stellung als in Westeuropa. Sie folgen, außer in Estland, dem deutschösterreichischen Modell und sind überall zumindest potenzielle Vetospieler.

Wie ein Vergleich der in den einzelnen Beiträgen unternommenen Erklärungen für die intra- und inter-(sub)regionalen Unterschiede zeigt, spielen für das institutionelle Design, zum Beispiel von Verfassungen, Wahlsystemen und Verfassungsgerichten, unmittelbar akteurszentrierte Faktoren, die unterschiedlichen Pfade der Systemtransformation und der Einfluss der EU im Beitrittsprozess eine wichtige Rolle, kaum jedoch „sozialistische Hinterlassenschaften“. Die Spezifik organisierter Interessen und Parteien sowie die reale Funktionsweise der politischen Institutionen lassen aber durchaus historische Prägungen erkennen. Dennoch scheint die unmittelbare Ursache für „MOE-Besonderheiten“ bei Parlamenten und Regierungen vorrangig in der insgesamt nachlassenden, noch immer aber hohen Dynamik der Parteiensysteme zu liegen, also womöglich stärker auf das geringe Alter der Parteiensysteme als auf legacies zurückzuführen zu sein.

Alle Beiträge des Sammelbands analysieren ihre Gegenstände mit dem Instrumentarium der jeweiligen Diskurse in der Vergleichenden Politikwissenschaft und argumentieren vom internationalen Forschungsstand aus. Zum einen stellen sie damit Politikwissenschaftlern ohne Regionalkenntnisse wertvolle Überblicke über die Verschiedenheit der jungen Demokratien zur Verfügung und tragen Erkenntnisse zusammen, die etablierte Diskussionen bereichern. Zum anderen erhalten Area-Spezialisten durch diese Beiträge knappe, disziplinierte Einführungen in systematische politikwissenschaftliche Diskurse, deren Relevanz für politische Dynamiken in der Region überzeugend demonstriert wird. Nicht zuletzt machen die Beiträge des Bandes mit ihren Befunden und Interpretationen auch deutlich, wie erfolgreich sich die Forschung zu MOE in den letzten Jahren in die Vergleichende Politikwissenschaft integriert hat. Dies ist zum Teil der disziplinären Entwicklung geschuldet, zum Teil aber auch dem Wandel innerhalb dieser Länder. Faktisch beschreiben die Autoren die schleichende Auflösung einer Subregion, die sich im Ergebnis der demokratischen Konsolidierung und EU-Europäisierung vollzieht, auch wenn sie von ihrem Vorgehen her an der Gegenüberstellung von „Mittel- und Ost“- sowie „West“-Europa festhalten und offenbar festhalten wollen.

In diesem Zusammenhang liegt eine Rückschau auf das „Dilemma der Gleichzeitigkeit“ marktwirtschaftlicher, demokratischer und nationalstaatlicher Reformen nahe, das Claus Offe und Jon Elster vor zwanzig Jahren formulierten. Seinerzeit lenkte es die Aufmerksamkeit darauf, dass die Etablierung von Demokratie kein Selbstläufer ist, wie Optimisten hofften. Wie relevant aber ist diese Gedankenfigur für die empirisch beobachtbaren Demokratisierungsprozesse tatsächlich gewesen? Wolfgang Merkels Zusammenschau zum Konsolidierungs- 
grad von Demokratien (ein faktischer Wiederabdruck aus PVS 3/2007) präsentiert die MOE-Erfolgsfälle als theoriewidrige Überraschung. Meines Erachtens steht seine These, das „puristisch“ handlungstheoretische „Dilemma der Gleichzeitigkeit“ sei damit widerlegt, aber auf wackligen Füßen. Laut Merkel habe dieses Theorem „deutlich weniger als 50 Prozent der Ergebnisse“ (S. 39) vorausgesagt. Dies trifft aber rechnerisch nur zu, weil lediglich 18 der 29 Länder ausgewählt werden, in denen der Staatssozialismus zusammengebrochen ist - die elf übrigen postsozialistischen Staaten werden nicht einbezogen; sie sind heute sämtlich nicht-demokratisch verfasst.

Wichtiger noch: Nimmt man die explizite Aufmerksamkeit für Kontingenz ernst, die den handlungstheoretischen Erklärungsansatz auszeichnet, kann das Dilemma-Theorem nicht mit dem ihm hier unterstellten starken Prognoseanspruch dahergekommen sein. Es postulierte die logische Unmöglichkeit, bestimmte Systemreformen gleichzeitig und endogen zum Erfolg zu führen, wenn Mechanismen der Konfliktbearbeitung, die Hinnahmebereitschaft der Transformationsverlierer und andere günstige Bedingungen nicht verfügbar wären. Wie gerade die zehn MOE-Länder zeigen, in denen das Dilemma zugunsten von Demokratie und Marktwirtschaft aufgelöst wurde, gab es in der Realität aber kaum logische Gleichzeitigkeit, hingegen viel politische Geduld, und der Demokratisierungserfolg war in einigen, für die Theorie relevanten Fällen entscheidend an starke externe Unterstützung gebunden. Dieses Argument durchzieht einige Beiträge des Sammelbands, für Frank Schimmelfennig und Guido Schwellnus ist es sogar zentral (explizit S. 288 f., S. 296). In diesem Sinne scheint Merkels These auch durch die vorliegenden empirischen Untersuchungen zumindest nicht gestärkt.

Als ganz besonderer Service für die Leser ist zu würdigen, dass der Band einen ausführlichen Materialanhang enthält, der die wichtigsten Daten für alle untersuchten Strukturdimensionen in allen zehn Ländern im Zeitraum von 1989/90 bis 2008 zur Verfügung stellt.

Petra Stykow

\section{Schriften von Hugo Preuß als entschiedene Verstärkung liberaler Gegenperspektiven}

Preuß, Hugo: Gesammelte Schriften. Zweiter Band: Öffentliches Recht und Rechtsphilosophie im Kaiserreich, herausgegeben und eingeleitet von Dian Schefold in Zusammenarbeit mit Christoph Müller, Tübingen 2009, 891 Seiten, € 99,-.

Das vorliegende Buch bietet ein Teilstück einer gar nicht hoch genug einzuschätzenden Gesamtedition, denn sie zeigt Hugo Preuß auf einer ersten Ebene als politischen Professor der Kaiserzeit par excellence und ist weiter geeignet, ihn als Urschöpfer ${ }^{1}$ der Weimarer Verfassung sowie seinen weniger bekannten Kampf um ihre Einhaltung verständlicher zu machen. Dabei handelt es sich, um den zuletzt veröffentlichten der fünfbändig angelegten Gesamtedition. Die beiden ersten nunmehr geschlossen vorliegenden Bände ${ }^{2}$ geben das

1 So Ernst von Hippel, Archiv für Rechts- und Wirtschaftsphilosophie, Bd. XXIV (1930/31), H. 4, S. 271.

2 Zusammen mit dem ersten Band: Politik und Gesellschaft im Kaiserreich, herausgegeben und eingeleitet von Lothar Albertin, Tübingen 2007. 